Original Paper http://ajol.info/index.php/ijbcs http://indexmedicus.afro.who.int

\title{
Régulation du comportement maternel chez l'araignée Coelotes terrestris (Agelenidae, Araneae) : études des éthogrammes relatifs aux interactions de confrontations femelle-jeunes conspécifiques
}

\author{
Bessekon Denis ASSI ${ }^{1 *}$, Koffi Mathias YAO ${ }^{1}$, Kouakou TIECOURA ${ }^{2}$ et \\ Kobenan Fiéni Jean Baptiste ADOU ${ }^{1}$ \\ ${ }^{1}$ Université Félix Houphouet-Boigny de Cocody, UFR Biosciences, \\ Laboratoire de Neurosciences, 22 B.P. 582 Abidjan 22, Côte d'Ivoire. \\ ${ }^{2}$ Université Félix Houphouet-Boigny de Cocody, Laboratoire de Génétique, \\ 22 B.P. 582 Abidjan 22, Côte d'Ivoire. \\ *Auteur correspondant ; E-mail: abdmaatre@gmail.com; Tel : +22505892430
}

\section{RESUME}

L'activité parentale, surtout maternelle et sa régulation ne sont pas l'apanage des seuls vertébrés. Chez les arachnides, les exemples abondent. C'est le cas de Coelotes terrestris, une araignée terricole de la famille des Agelenidae qui nourrit ses petits pendant au moins quatre semaines. Mais compte tenu des difficultés d'observation en milieu naturel, une étude expérimentale a été menée en laboratoire dans le but de comprendre les interactions entre la femelle et ses jeunes. Ainsi, cinq catégories de femelles ont été mises en confrontations forcées avec des jeunes homospécifiques. Les interactions de ces confrontations ont été enregistrées et analysées. Les résultats montrent qu'en présence des jeunes, l'expression des «sursauts» (SST) traduit vraisemblablement une attitude agressive des femelles vis-à-vis des jeunes car, ce sont les femelles en préémergence (pré-ponte, incubante et sub-adulte) connues pour être agressives ou "tueuses" de jeunes qui présentent significativement la manifestation de ce comportement. Par contre, l'activité de «tissage » (TIS) peut être qualifiée de «comportement maternel». Son expression semble traduire une certaine tolérance et/ou reconnaissance des femelles à l'égard des jeunes. En effet, ce sont les femelles en post-émergence (nourricière et post-dispersion) déjà montrées comme les plus tolérantes qui posent significativement cet acte (de tissage).

(C) 2015 International Formulae Group. All rights reserved.

Mots clés : Interaction, agressivité, sursaut, tolérance, tissage, comportement maternel.

\section{INTRODUCTION}

Généralement, lorsqu'on parle de comportement parental, les vertébrés servent presque systématiquement de référence et l'on accorde très peu d'importance aux arthropodes. Chez ces derniers les exemples d'activité parentale abondent pourtant, non seulement chez les insectes (Billick et al., 2001; Park et Choe, 2003a ; Park et Choe, 2003b), mais aussi dans d'autres groupes comme les arachnides (Assi et Trabalon, 2005 ; Salomon et al., 2005; Viera et al., 2006 ; Viera et Romero, 2008; Stefani et al., 2011a ; Yip et Rayor, 2014). La conséquence d'une telle vision non justifiée est qu'elle occulte chez les arachnides certains aspects essentiels de la vie de relation des animaux. Il s'agit des aspects liés justement à la régulation 
du comportement parental basée sur les échanges interindividuels de reconnaissance et de tolérance réciproque ainsi que des échanges entre l'individu et son milieu. Ces aspects sont ainsi assimilés à ce qui est communément appelé les «complexes instinctifs » (Grasse, 1942; Deleurance, 1957; Gervet, 1964a), c'est-à-dire un ensemble d'actes spécifiques se déroulant de façon aveugle et strictement programmés qui contribue à la réalisation d'une fonction biologique précise. Or, l'automatisme génétique est incompatible avec la régulation.

Chez Coelotes terrestris, après l'émergence, les jeunes restent groupés dans le tube de la toile maternelle et ne se dispersent qu'au bout de quatre à cinq semaines plus tard (Tretzel, 1961a, 1961b; Gundermann et al., 1989). Cette situation grégaire nécessite non seulement des échanges et interactions mèrejeunes et jeunes-jeunes, mais également des mécanismes de régulation adaptés à cette activité maternelle. Or, C. terrestris, espèce d'araignée terricole vit dans des anfractuosités ou sous les pierres; d'où la difficulté ou l'impossibilité d'observer, en milieu naturel, ces interactions. Ainsi, pour mieux contrôler et suivre les échanges entre les femelles et les jeunes, nous avons opté pour une étude des interactions provoquées expérimentalement en laboratoire.

Les études antérieures ont montré que pendant les cinq premières minutes de confrontation, les femelles pré-pontes et incubantes provoquent un taux de mortalité plus élevé des jeunes et qui s'amplifie après vingt-quatre heures alors que ce taux reste très faible, voire nul chez les femelles nourricières et post-dispersion (Assi et Horel, 1996; Assi, 1997).

Dans la présente étude, il s'agit de déterminer la nature des interactions entre les femelles et les jeunes et d'établir si possible le lien entre le comportement des femelles et ses conséquences sur les jeunes. En d'autres termes, il s'agit d'identifier, d'une part, les comportements de la femelle qui conduisent à l'agression et à la mise à mort des jeunes et, d'autre part, les comportements associés à la tolérance.

Etant donné la rapidité avec laquelle les femelles de $C$. terrestris réagissent face à un quelconque objet entrant en contact avec leur structure soyeuse, l'identification de cet objet devrait également se faire rapidement. C'est la courte phase pendant laquelle les choses semblent se décider rapidement qui paraît la plus intéressante afin de voir si dans un laps de temps aussi court que cinq minutes d'interactions, des différences comportementales pouvaient apparaître entre les différentes catégories de femelles.

\section{MATERIEL ET METHODES}

\section{Matériel biologique}

Coelotes terrestris est une araignée terricole européenne vivant sous les pierres ou dans diverses anfractuosités. C'est une espèce sub-sociale présentant successivement une phase de soins au cocon et une phase de soins aux jeunes. Cette dernière phase dure quatre à cinq semaines pendant laquelle la mère et ses jeunes vivent ensemble dans le nid maternel en développant des interactions de type social. La mère assure donc l'alimentation de sa portée, notamment par des pontes trophiques et par des proies capturées qu'elle leur abandonne. Au terme des cinq semaines, les jeunes se dispersent et chacun mène une vie solitaire.

\section{Dispositif d'observation}

Cinq catégories de femelles ont été constituées:

- les sub-adultes (SUB) issues de l'élevage de juvéniles récoltés dans la nature au cinquième ou sixième stade nymphal, $\mathrm{N}=9$;

- $\quad$ les pré-pontes (PRE), femelles adultes fécondées dans la nature qui n'avaient pas encore pondu de cocon au moment de l'expérience; c'est à partir de leur élevage en laboratoire que les autres femelles et les jeunes sont obtenus, $\mathrm{N}=15$; 
- $\quad$ les incubantes (INC) étaient en train de garder leur premier cocon, $\mathrm{N}=17$; les nourricières (NOU) avaient préalablement pondu au laboratoire et en étaient à soigner leurs petits au moment de l'expérience, $\mathrm{N}=18$;

- $\quad$ les post-dispersions (POS) qui avaient élevé leurs petits au laboratoire et avaient été maintenues en isolement pendant un mois après la dispersion de leurs jeunes, $\mathrm{N}=9$.

Les femelles $C$. terrestris qui, par rapport au cycle de reproduction, sont positionnées avant l'émergence des jeunes sont dites femelles en pré-émergence (SUB, PRE, INC); celles qui se situent après l'émergence des jeunes sont qualifiées de post-émergence (NOU, POS).

Pour réaliser les tests de confrontations, des jeunes actifs âgés de trois semaines ont été utilisés. Le principe des tests de confrontation est celui décrit par Assi et al. (1992) et Assi et Horel (1996). Il consiste à introduire un groupe de jeunes chez une femelle et à suivre pendant un temps de confrontation les interactions qui en découlent.

$\mathrm{Au}$ moment des tests, chaque femelle des cinq catégories a reçu un groupe de dix jeunes étrangers tous issus d'une même portée. D'abord contenus dans un tube à essai, les jeunes ont tous été introduits sans ménagement sur la toile de la femelle. Les interactions qui découlent des cinq premières minutes de ces confrontations forcées étaient enregistrées par un système vidéoscopique (Figure 1). L'analyse des enregistrements a permis de dresser une liste d'items comportementaux.

\section{Les items comportementaux}

Dix-sept items comportementaux identifiables exprimés au cours des interactions ont été répertoriés (Tableau 1). Pendant les cinq premières minutes de confrontation, la fréquence de mise à mort des jeunes est si faible qu'aucun item n'a été retenu pour la mise à mort.

\section{Traitement des données et analyses statistiques \\ Analyse des éthogrammes}

Pour avoir une vue globale des différents comportements exprimés, un diagramme de succession d'items comportementaux a été réalisé selon le principe de l'analyse transitionnelle (Chatfield et Lemmon, 1970; Gervet et Truc, 1976), pour chaque catégorie de femelle. Selon cette analyse, les différents actes des femelles sont chiffrés dans une matrice de succession qui est un tableau à double entrée portant en abscisses l'ensemble des actes antérieurs, en ordonnées l'ensemble des actes ultérieurs. On peut donc dresser à partir de cette matrice un diagramme de flux dans lequel chaque acte antérieur est relié à un acte ultérieur par une flèche dont l'épaisseur est proportionnelle à la fréquence des successions observées. De même, la surface occupée par un acte est proportionnelle à la fréquence relative de chaque acte (Chatfield et Lemmon, 1970). Par souci de clarté, seules les successions différentes du hasard sont représentées $\left(\mathrm{X}^{2}\right.$ : $\mathrm{P}<0,05)$; les actes des jeunes ne sont pas représentés.

\section{Analyse des profils comportementaux}

Après avoir relevé et dénombré les occurrences des items comportementaux, on peut calculer les pourcentages moyens d'occurrences de ces items pour chaque catégorie de femelles au cours des cinq minutes d'interaction.

\section{Analyse hiérarchique ascendante et de classification}

La technique de l'analyse hiérarchique (Gruvaeus et Wainer, 1972; Hartigan, 1975), comme les diagrammes de flux et les profils, est une technique qui donne une vue globale des catégories et permet de comparer les données selon des critères différents. En l'appliquant à l'ensemble des catégories, on cherche à voir quelles sont les plus proches ou les plus éloignées les unes des autres sur le plan des réponses comportementales au cours des interactions. Au niveau des actes, l'analyse permettra également de les classer en fonction 
de l'importance de leur expression et des rapprochements qui en découlent entre les catégories de femelles.

\section{Tests de Kruskal-Wallis et de Mann- Whitney}

Dans le cadre de l'analyse de l'évolution temporelle des «sursauts » (SST) et du «tissage» (TIS), deux méthodes de statistiques non paramétriques ont été utilisées: le test de Kruskal-Wallis pour l'hétérogénéité globale et le test U de MannWhitney pour les comparaisons a posteriori deux par deux des catégories de femelles.

La présente étude a été menée en deux temps : - dans un premier temps, il s'agissait de décrire les principales caractéristiques des interactions entre les femelles et les jeunes (étude descriptive);

- dans un second temps, il s'agissait d'affiner les analyses comportementales afin de détecter les corrélations éventuelles entre l'attitude ou les réponses des femelles et les types d'interactions qu'elles développent avec les jeunes et leurs conséquences (étude explicative).

\section{RESULTATS}

Etude descriptive des comportements exprimés par les femelles

Ethogrammes des femelles confrontées aux jeunes

Pendant les cinq minutes d'observation directe en l'absence de jeunes, l'activité motrice des femelles, quelle que soit la catégorie, est nulle. On peut en déduire que tous les actes exprimés par chaque femelle après l'introduction des jeunes sont dus à la présence de ces derniers.

Les diagrammes de flux de la Figure 2 diffèrent nettement selon les catégories de femelles: on trouve les plus simples chez les sub-adultes (SUB) et les post-dispersions (POS) ; ils traduisent un nombre réduit d'items ainsi que de liaisons très peu significatives. Chez les autres catégories, le problème est de savoir si la complexité apparente des flux tient à la complexité des interactions ou à la variabilité interindividuelle des femelles. En effet, on note que chez les incubantes (INC), les successions sont denses et complexes. Ceci semble témoigner d'une forte activité motrice des femelles dominée par les «sursauts » (SST). Ce même type d'interactions se retrouve presque chez les femelles pré-ponte (PRE), mais marqué par une différence majeure, qui est l'absence de tissage (TIS). Les nourricières (NOU) présentent presque le même type de complexité des interactions avec, comme principale activité, le tissage. Cependant les femelles SUB se rapprochent des femelles PRE et des INC par leur forte activité de «sursauts» (SST); alors que les femelles POS sont plus proches des NOU par l'importance de leur activité de tissage.

Chez toutes les catégories de femelles, l'approche rapide (APR) est généralement suivie du contact (COS), et souvent du tapotement (TAP). Mais pour les femelles INC et PRE, il se dégage un axe principal des successions: (APR)-(COS)-(TAP)-(SAC)(TRA).

\section{Description des rencontres}

La chute des jeunes sur la toile de la femelle provoque instantanément une réaction de celle-ci qui se traduit par une «approche rapide» (APR) suivie le plus souvent d'un « contact» (COS). L'approche de la femelle entraîne la «fuite » (FUI) des jeunes. La suite des événements va dépendre des catégories de femelles. On peut décrire deux types de situations: les rencontres «violentes » et les rencontres « pacifiques ».

\section{Les rencontres « violentes »}

Dans cette situation les jeunes contactés (COS) par les femelles sont tapotés (TAP) plusieurs fois à l'aide des pattes antérieures et des pédipalpes. Les tapotements sont suivis de sursauts (SST). Ces mouvements qui persistent bien après l'arrêt des ébranlements provoqués à l'origine par le(s) jeune(s), entraînent la fuite (FUI) de ceux-ci, même lorsqu'ils sont à distance de la 
femelle. La fuite sera d'autant plus accentuée que les sursauts persisteront.

Les tapotements (TAP) s'effectuent généralement sur l'abdomen des jeunes. Il s'ensuit un glissement (GLI) de ces derniers, soit en avant de la femelle, soit en arrière sous elle, ce qui oblige parfois la femelle à reculer et à faire demi-tour (DET).

La saisie d'un jeune dans les chélicères (SAC) intervient en dehors des moments d'interactions brutales. Les chélicères se rouvrent au moindre mouvement du jeune, faisant échapper certains jeunes facilement de la bouche de la femelle. Toutefois, quand un jeune résiste au transport en s'accrochant énergiquement au substrat, une lutte s'engage entre lui et la femelle qui persiste à le déplacer. Dans ces conditions, des blessures plus ou moins graves sont infligées au jeune en question avec plus ou moins de coulées d'hémolymphe au niveau de la zone saisie par les chélicères. Le jeune saisi est ensuite transporté (TRA) et déposé (DPO) en des endroits privilégiés, notamment au fond de la galerie, ou dans les angles intérieurs de la boîte d'expérimentation. Certaines femelles rangent les jeunes saisis du revers des pédipalpes et repartent aussitôt. Des transports répétés de jeunes peuvent se manifester pendant les cinq minutes d'observation, voire au-delà. Lorsqu'ils sont intacts, les jeunes déposés reviennent dans la galerie. Ceux qui sont gravement blessés sont retrouvés morts le lendemain, abandonnés sur place.

\section{Les rencontres « pacifiques »}

Les rencontres pacifiques sont dominées par une forte activité de tissage (TIS) des femelles. Par contre l'activité de sursaut (SST) est presque nulle. Le tissage intervient après que chacune des femelles a été en contact physique avec au moins un jeune. Lorsque les jeunes sont contactés (COS) ou tapotés (TAP), ces derniers s'immobilisent (IMO). Cette immobilisation semble inhiber toute attitude agressive de la part de la femelle qui les abandonne. Avec le temps, les contacts directs initiés par les jeunes en direction des femelles deviennent de plus en plus fréquents et ne suscitent aucune attitude négative des femelles à leur encontre.

Les rencontres violentes sont observées essentiellement chez les femelles en préémergence et plus particulièrement les incubantes et pré-ponte. Alors que les rencontres «pacifiques » sont caractéristiques des interactions des femelles en postémergence.

\section{Les profils comportementaux Fréquence relative des items}

L'examen des profils qui découle de l'analyse statistiques des items de la Figure 3, confirme bien que les femelles peuvent être rangées en deux grands groupes: d'un côté les femelles en pré-émergence composées des femelles SUB, PRE et INC exprimant plus les «sursauts » (SST) et de l'autre, les femelles en post-émergence comprenant les NOU et POS qui manifestent plus les « tissage » (TIS).

\section{Analyse hiérarchique des items des catégories de femelles}

Les résultats (Figure 4) montrent deux groupes essentiels: les femelles en préémergence parmi lesquelles on note un plus grand rapprochement des femelles sub-adulte, pré-ponte et incubante; les femelles en postémergence avec les femelles nourricières rattachées aux femelles post-dispersion.

$\mathrm{Au}$ niveau des actes exprimés (Figure 5), on note une prédominance de l'expression du tissage (TIS), nettement détaché du reste des actes. Ensuite, viennent deux grands groupes d'actes dont l'un contient les actes allant de déplacement (DEP) à sursaut (SST) (G1). L'autre groupe contient les actes allant de fuite (FUI) à approche rapide (APR) (G2). La subdivision ultérieure isole le (SST) en tant que deuxième principal acte exprimé suivi du tapotement (TAP). Les autres actes restants semblent présenter une expression d'importance relativement identique.

Pour mieux comprendre les différentes attitudes comportementales, il faut maintenant analyser un peu plus en détail certains items 
comportementaux caractéristiques de ces interactions. Le choix des actes est fait, en partie, à partir de l'analyse hiérarchique qui permet de les séparer en fonction de leur importance quantitative: c'est le cas de sursaut (SST) et de tissage (TIS). D'autres sont choisis en fonction de leur importance qualitative: c'est le cas de tapotement (TAP) et contact simple (COS) ou encore en fonction de leur particularité: tel est le cas de transport (TRA).

Etude explicative des comportements exprimés par les femelles Le transport des jeunes

En dépit du fait que le comportement de transport (TRA) n'est pas le plus fréquent, on s'est intéressé à la particularité de cet acte en raison de son aspect surprenant. En effet, généralement ce sont les proies qui sont saisies dans les chélicères pour être transportées à l'intérieur du tube de l'Araignée et y être consommées. Or, dans le cas de la présente étude, les jeunes vont être transportés à l'intérieur du tube de la femelle non pas pour être mangés mais plutôt pour être déposés, morts ou vivants, dans un coin de la boîte, en l'occurrence le fond de la galerie qui correspond généralement au lieu de dépôt de restes non ingérés de proies.

Les résultats de la Figure 6 représentent les pourcentages de femelles ayant transporté au moins un jeune pendant les cinq minutes d'interaction. On remarque que les femelles qui transportent le plus les jeunes sont les femelles INC $(60 \%)$ suivies des femelles PRE $(35 \%)$ et des femelles SUB (22\%). L'expression de cet item reste marginale chez les femelles NOU (seules 2 sur 18 l'ont manifesté); aucune des femelles POS n'a montré cette activité. Ces résultats montrent clairement que les femelles impliquées dans l'expression de l'acte «transport de jeunes» (TRA) sont celles qui n'ont pas encore de jeunes; ce sont aussi les femelles qui, dans leur comportement, vont jusqu'à la « saisie des jeunes dans leurs chélicères » (SAC). De ce fait, les femelles qui n'ont pas exprimé l'acte
(SAC) telles que les femelles POS, n'ont pas non plus manifesté tous les actes subséquents logiquement liés à l'expression de cet acte, à savoir: «transport des jeunes» (TRA), « dépôt » (DPO), « relâche le jeune » (REL).

Pour mieux comprendre dans quelles circonstances apparaissent les actes «saisie » et «transport» lors des interactions, on a analysé l'expression de deux actes importants non seulement pour leur fréquence, mais aussi par leurs caractéristiques traduisant vraisemblablement le type d'interactions susceptibles d'être rencontrées: «violentes » ou « pacifiques ».

\section{«Sursauts » et « Tissage »}

$\mathrm{Au}$ vu des résultats de la Figure 3, les catégories de femelles ont été séparées en femelles exprimant plus les «sursauts » $(\mathrm{SST})$ et en femelles exprimant plus le «tissage » (TIS). Ainsi, Les fréquences relatives de chacun des deux actes ont été relevées minute par minute pour suivre leur évolution au cours des interactions.

\section{Les « sursauts » $(\mathrm{SST})$}

Les résultats montrent que chez les femelles en pré-émergence et plus précisément les femelles PRE et INC, malgré la diminution des fréquences relatives de l'expression de cet acte au cours du temps, il y a une persistance de l'activité de sursauts (Figure 7). Cette activité reste presque nulle pour les femelles en post-émergence. En fait, ces différences n'apparaissent pas immédiatement. Le test de Kruskal-Wallis réalisé au bout d'une minute d'interaction ne montre pas d'hétérogénéité d'ensemble. Alors que de la deuxième à la cinquième minute d'interaction, les résultats montrent une hétérogénéité globale entre les différentes catégories de femelles (Tableau 2).

Les comparaisons a posteriori par le test U de Mann-Whitney (Tableau 3), entre les catégories de femelles deux par deux, montrent une différence significative entre femelles PRE et femelles INC au bout de deux et trois minutes d'interactions. A partir de la 
quatrième minute, aucune différence significative n'est mise en évidence entre ces deux catégories de femelles. Par contre, des différences très significatives apparaissent entre les femelles PRE et INC et les femelles en post-émergence. L'absence de différence entre femelles INC et PRE d'un côté, entre femelles NOU et POS de l'autre, pourrait traduire une certaine similitude dans l'expression des SST pour l'un ou l'autre couple de catégories de femelles.

Les femelles SUB étant classées parmi les femelles en pré-émergence, on peut toutefois noter une certaine ambiguïté pour ces femelles qui présentent des différences significatives avec les femelles INC et NOU, mais pas avec les femelles PRE et POS.

\section{Le « Tissage » (TIS)}

Au cours des interactions, les femelles SUB et PRE n'ont manifesté à aucun moment d'activité de tissage. L'expression de ce comportement est le seul fait de quelques femelles INC (4 femelles sur 17 testées) et de la quasi-totalité des femelles NOU et POS. De ce fait, les tests statistiques réalisés ici sont faits avec ces trois catégories de femelles.

Les résultats de la Figure 8 montrent que chez les femelles en post-émergence, la disparition rapide de l'activité de «sursauts» (SST) fait place progressivement à l'activité de «tissage » (TIS). Par contre, le niveau de tissage chez les femelles incubantes reste nul tout au long des cinq minutes d'interactions. Cependant, l'analyse statistique, test de Kruskal-Wallis du Tableau 3 ne montre pas d'hétérogénéité d'ensemble au bout d'une minute pour les trois catégories de femelles INC, NOU et POS. Alors qu'au-delà, à partir de la deuxième minute, on obtient une hétérogénéité d'ensemble au cours du temps. Les comparaisons, a posteriori, entre les catégories de femelles par le test U de MannWhitney (Tableau 4), montrent une apparition plus tardive d'une importante activité de tissage chez les femelles NOU. En revanche, cette activité est significativement plus importante et constante chez les femelles POS que chez les femelles NOU.

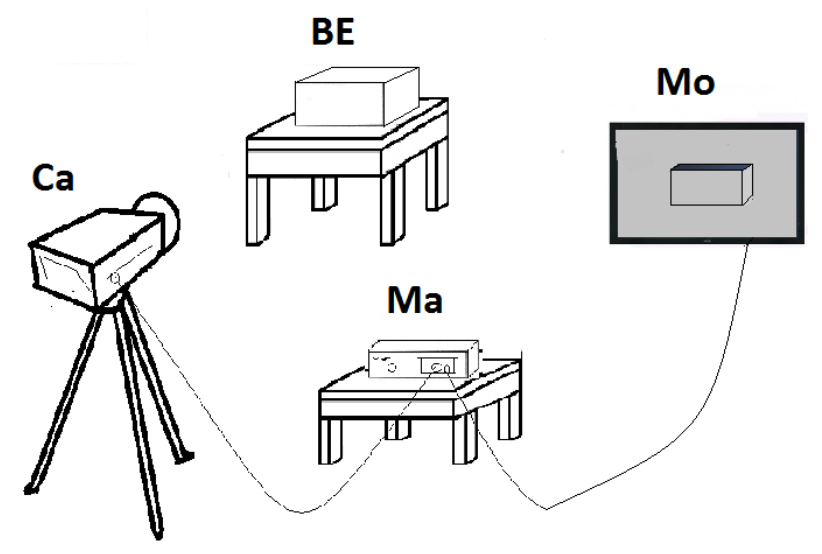

Figure 1: Schéma du dispositif expérimental vidéoscopique ; $\mathrm{BE}=$ boîte d'expérimentation; $\mathrm{Ca}=$ caméra; $\mathrm{Ma}=$ magnétoscope $\mathrm{Mo}=$ moniteur 


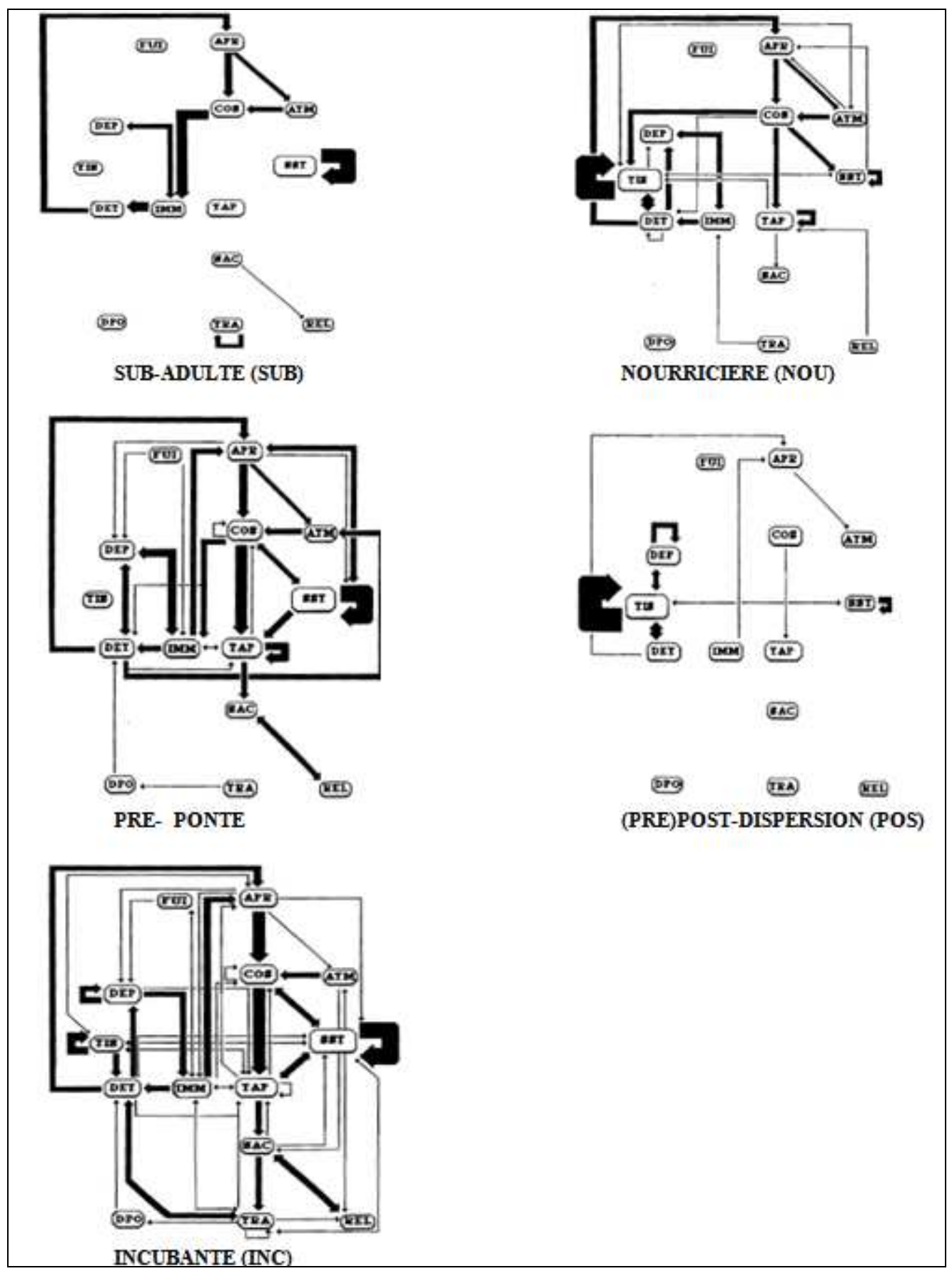

Figure 2: Diagrammes de succession des actes exprimés par les catégories de femelles au cours des cinq minutes d'interactions avec les jeunes. Seuls les enchaînements différents du hasard $\left(\mathrm{X}^{2}: \mathrm{P}<\right.$ $0,05)$ sont représentés. 


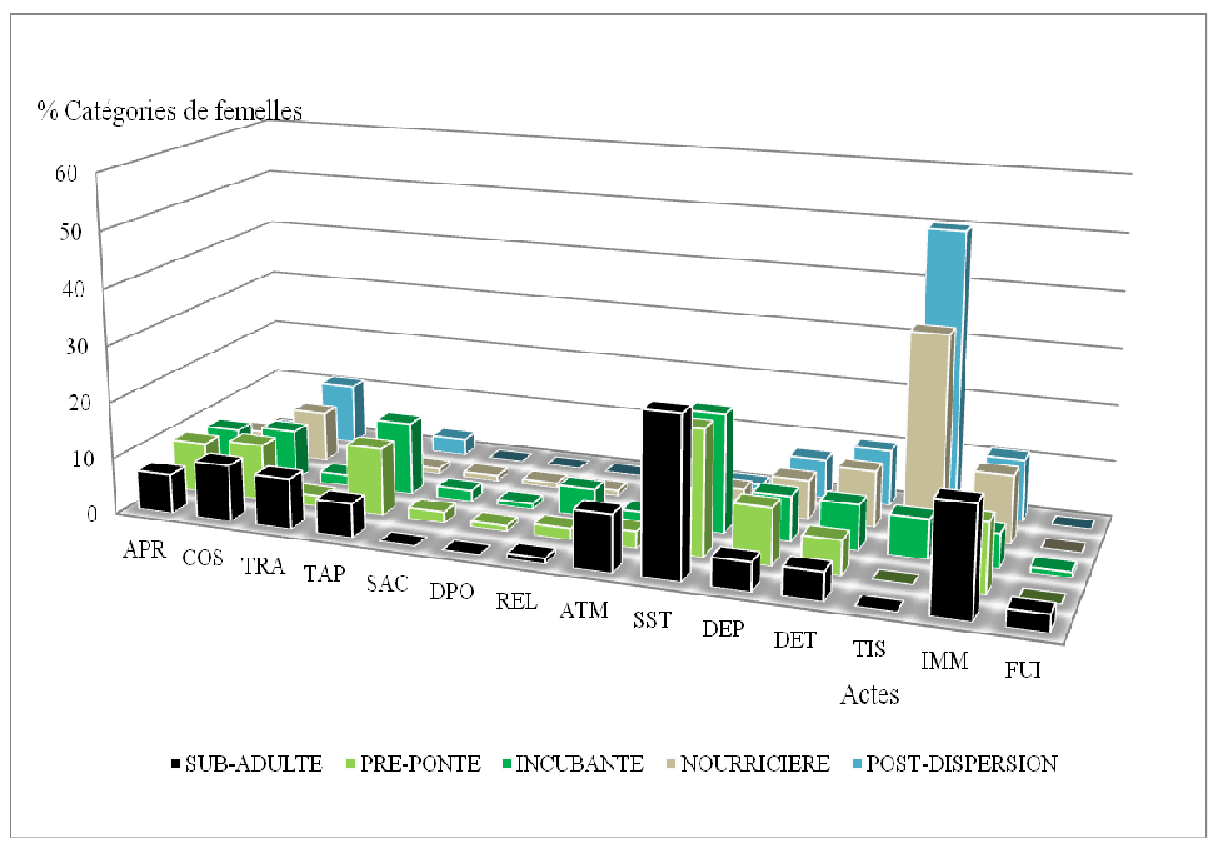

Figure 3: Histogramme des profils comportementaux (\%) des différentes catégories de femelles au cours des cinq minutes d'interactions.

\section{DISTANCES}

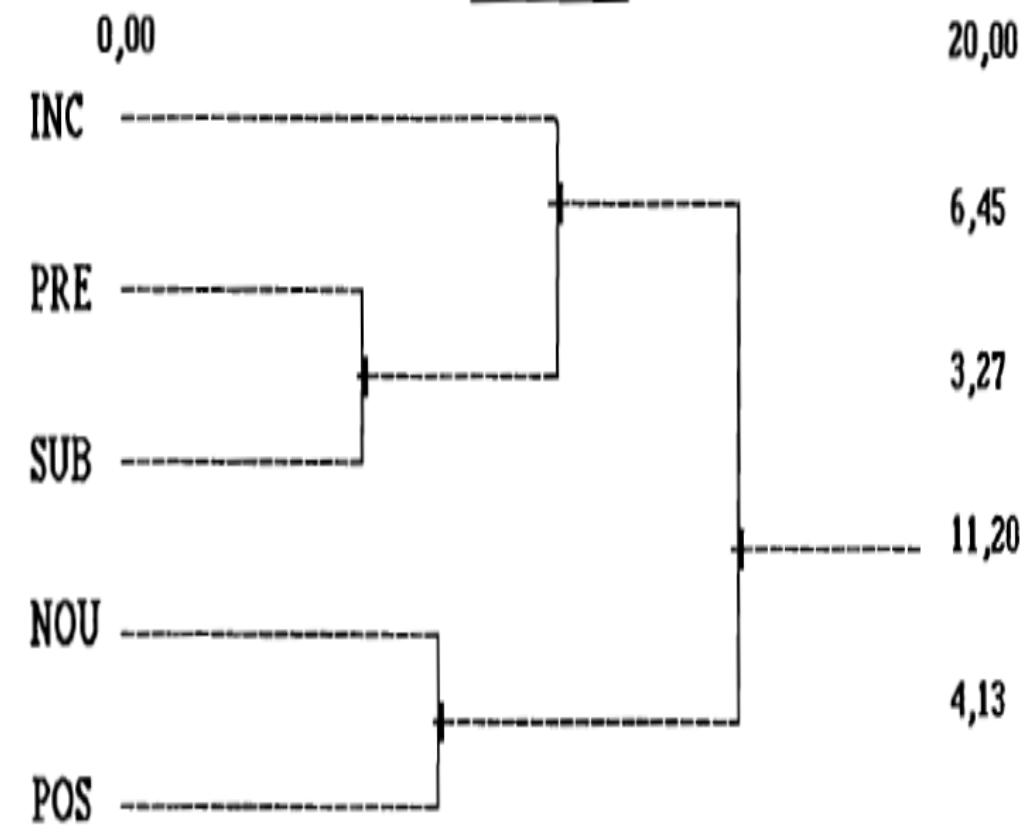

Figure 4: Diagramme en arbre du comportement des catégories de femelles. 


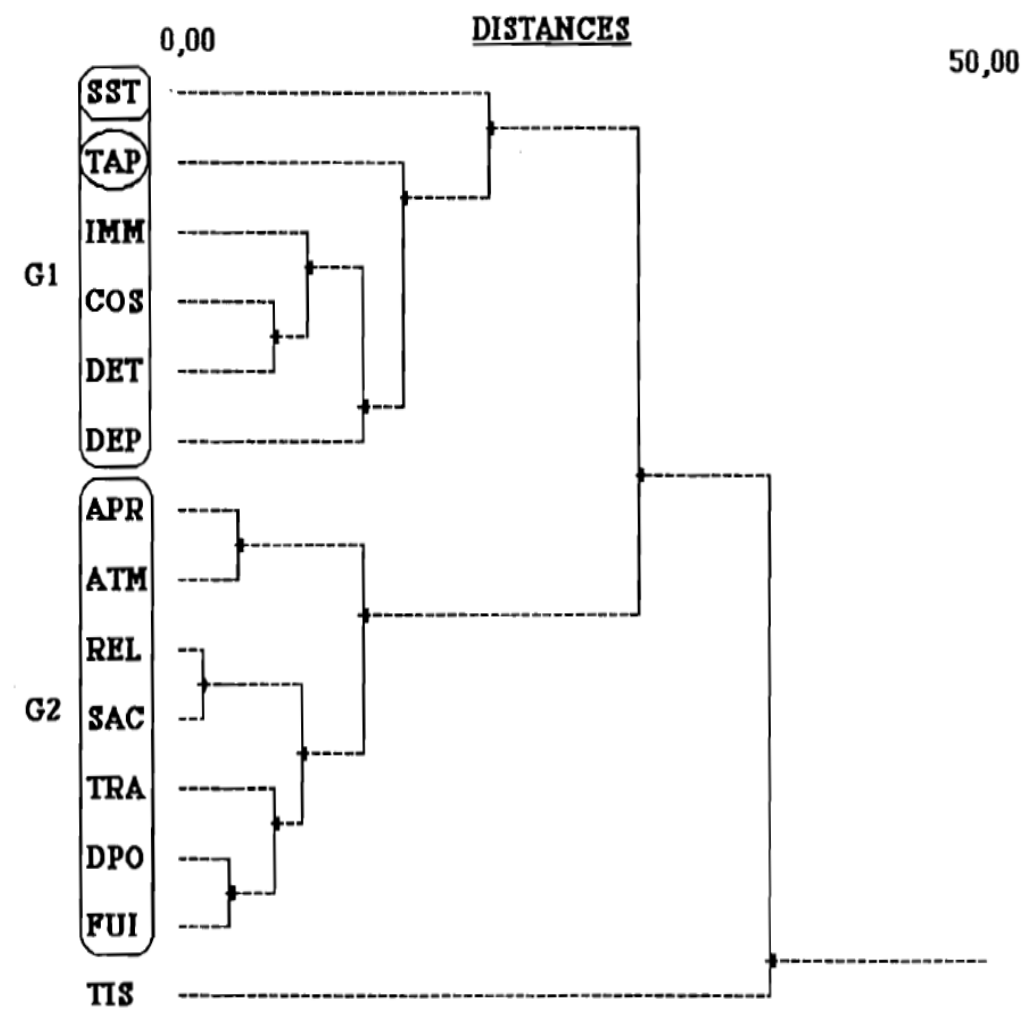

Figure 5: Diagramme en arbre des actes exprimés par rapport à l'ensemble des catégories de femelles.

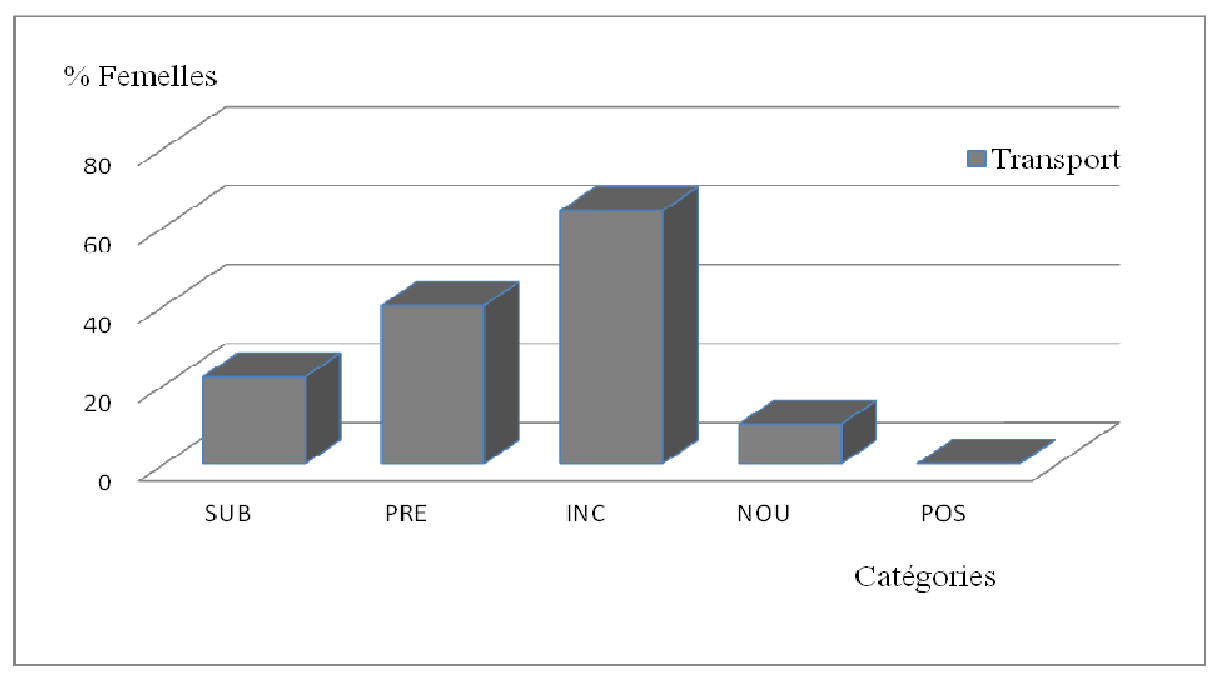

Figure 6: Pourcentage de femelles ayant transporté au moins un jeune au cours des cinq minutes d'interaction. 
8UB-ADULTE

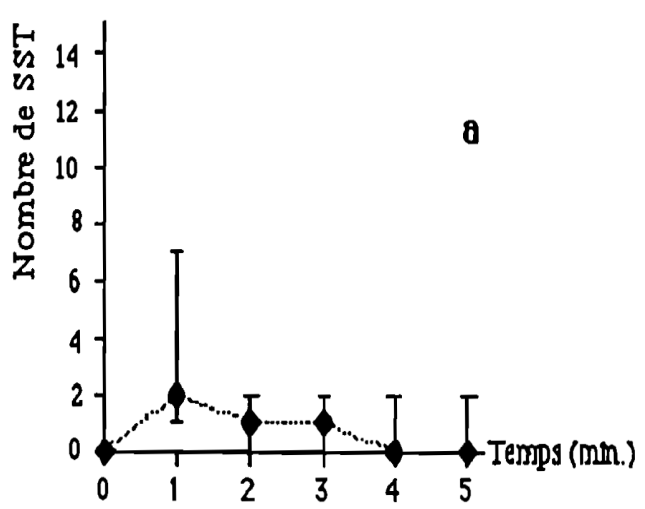

PRE-PONIE

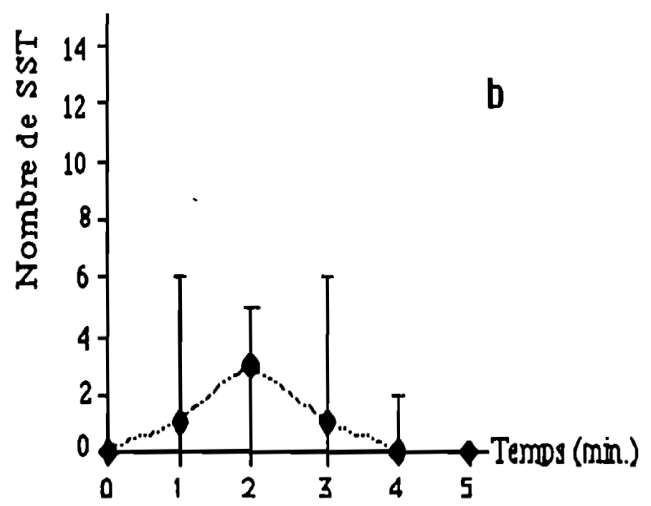

\section{INCUBAKIE}

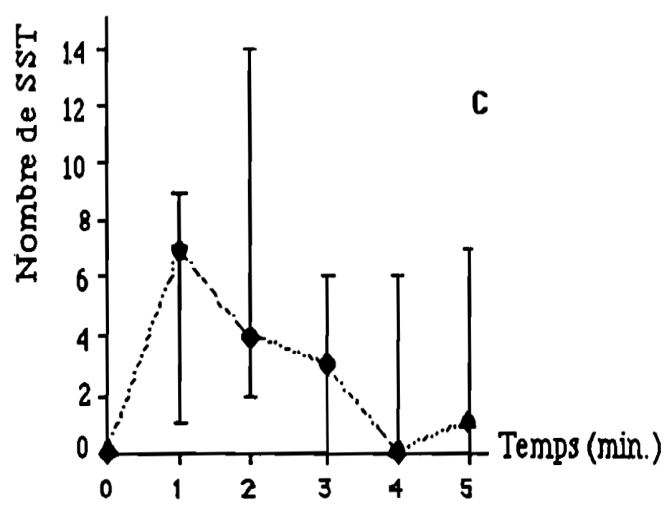

HOURRICIERE

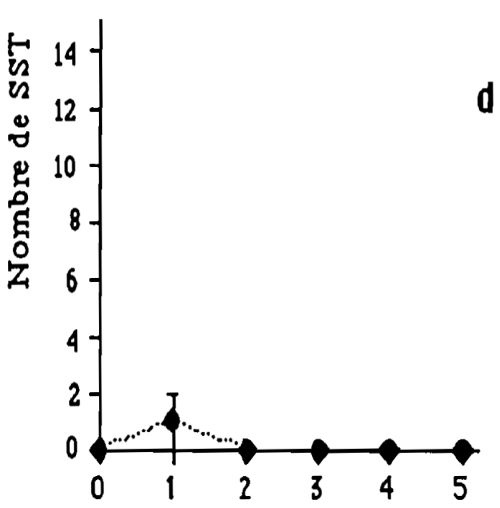

POET-DIRPER8IOH

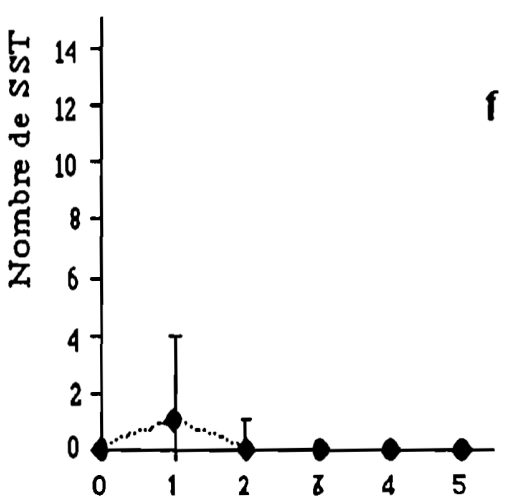

Figures 7: Evolution temporelle de l'expression des sursauts (SST) (médianes et quartiles). 


\section{DYCUBANTE}
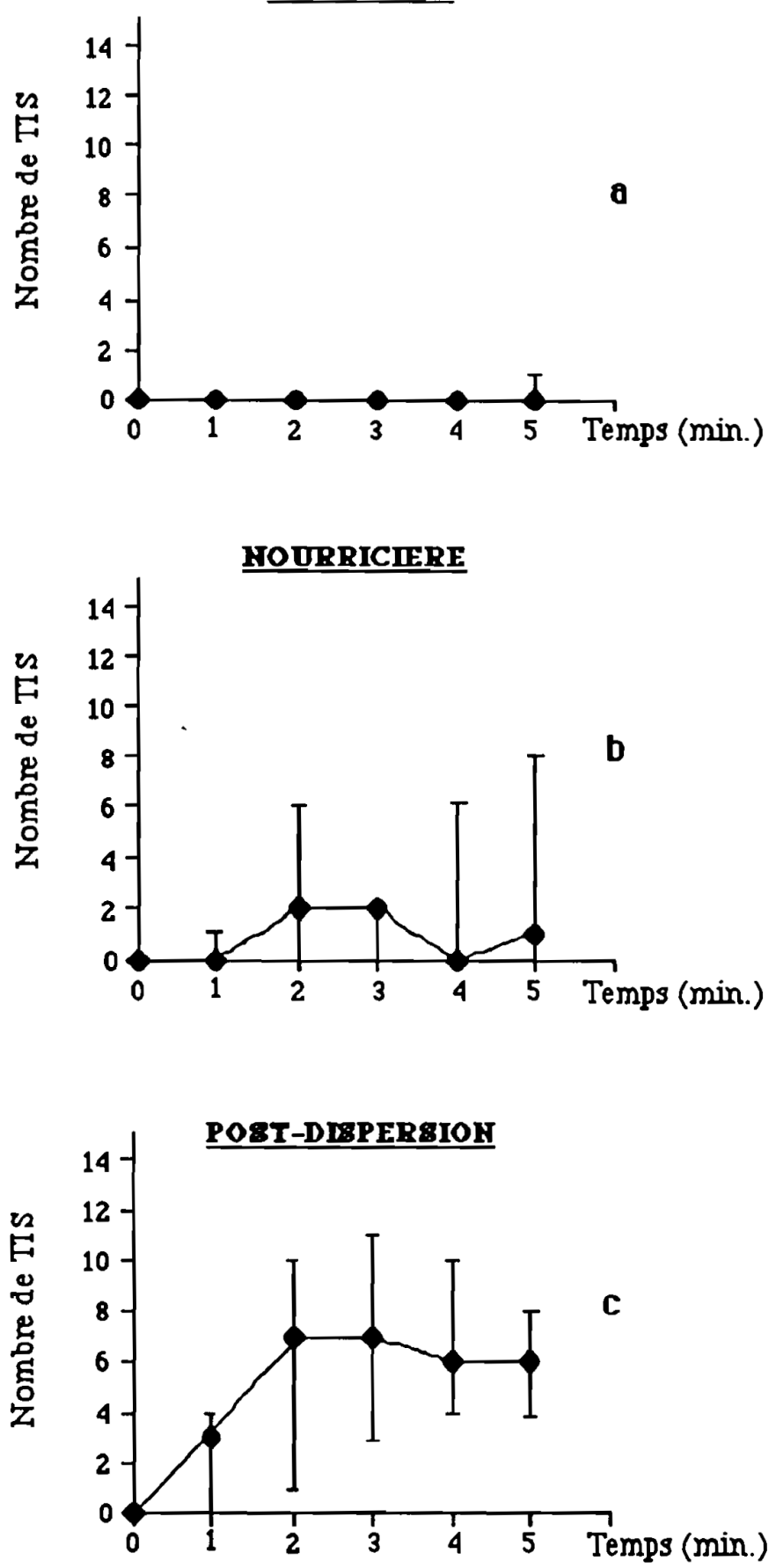

Figure 8: Evolution temporelle du tissage (médianes, quartiles) chez les femelles. 
Tableau 1: Définition des items comportementaux.

\begin{tabular}{|c|c|}
\hline Items & Signification \\
\hline it01 APR: & «APPROCHE RAPIDE »; déplacement rapide orienté vers les jeunes. \\
\hline it02 COS: & $\begin{array}{l}\text { «CONTACT SIMPLE »; au moins une des pattes antérieures de l'araignée se pose } \\
\text { sur le corps du protagoniste. }(* *)\end{array}$ \\
\hline it03 TAP: & $\begin{array}{l}\text { "TAPOTEMENT »; contact suivi d'une manipulation des jeunes à l'aide des pattes } \\
\text { antérieures et surtout des pédipalpes. }\end{array}$ \\
\hline it04 SAC: & « Saisie d’un jeune dans les chélicères ». \\
\hline it05 TRA: & $\begin{array}{l}\text { «TRANSPORT »; saisi dans les chélicères, le jeune est transporté pour être déposé } \\
\text { hors du tube soyeux. Le transport peut se faire sur plusieurs allers et retours. }\end{array}$ \\
\hline $\begin{array}{l}\text { it06 DPO: } \\
\text { it07 REL: }\end{array}$ & $\begin{array}{l}\text { «DEPOT » le jeune est déposé (en certains points précis). } \\
\text { «RELACHE LE JEUNE »; ouverture des chélicères qui libèrent le jeune. }\end{array}$ \\
\hline it08 ATM: & $\begin{array}{l}\text { «ATTAQUE-MENACE »; pour simplifier, un seul terme est employé qui } \\
\text { correspond toujours à la manifestation d'une agressivité à l'égard des jeunes (pour } \\
\text { MEN: «MENACE », la femelle frappe la toile avec ses premières paires de pattes, ce } \\
\text { qui fait fuir les jeunes ; «ATTAQUE », la femelle frappe le jeune à l'aide de ses } \\
\text { pattes antérieures). }\end{array}$ \\
\hline it09 SST: & $\begin{array}{l}\text { «SURSAUTS »; ce sont des mouvements synchronisés verticaux des pattes } \\
\text { antérieures et/ou des pédipalpes, manifestés en présence des jeunes (en fuite, ou au } \\
\text { cours d'interactions brutales). }\end{array}$ \\
\hline it10 DEP : & «DEPLACEMENT », une locomotion banale. $(* *)$ \\
\hline it11 DET : & «DEMI-TOUR » $(* *)$ \\
\hline it12 TIS : & « TISSAGE »; émission de soie non liée à la prédation. \\
\hline it13 FUI : & « FUITE »; l'individu s'éloigne rapidement. $(* *)$ \\
\hline it14 IMM : & «IMMOBILITE »; inactivité pendant 5 secondes au moins. (**) \\
\hline it15 GLI : & «SE GLISSE SOUS LA FEMELLE » (*). \\
\hline 16 GRI : & «GRIMPE SUR LA FEMELLE » (*). \\
\hline it17 IMO : & «IMMOBILISATION »; immobilité du jeune contacté par la femelle. $(*)$ \\
\hline
\end{tabular}

Tableau 2: Tests de Kruskal-Wallis de l'évolution temporelle des «sursauts » (SST) pour les cinq catégories de femelles pendant cinq minutes de confrontation.

\begin{tabular}{lccccc}
\hline Temps & $\mathbf{1 ~} \mathbf{~ m n}$ & $\mathbf{2} \mathbf{~ m n}$ & $\mathbf{3} \mathbf{~ m n}$ & $\mathbf{4} \mathbf{~ m n}$ & $\mathbf{5} \mathbf{~ m n}$ \\
\hline 4ddl $\mathrm{H}$ & 5,17 & 2,83 & 25,2 & 27,15 & 33,9 \\
Sig & $\mathrm{NS}$ & $\mathrm{P}=0,0004$ & $\mathrm{P}=0,0002$ & $\mathrm{P}=0,0002$ & $\mathrm{P}=0,0002$ \\
\hline $\begin{array}{l}\text { Sig.= significativité; ddl= degré de liberté; } \mathrm{H}=\text { valeur servant à calculer la probabilité p du test de Kruskal-Wallis; NS= non } \\
\text { significatif. }\end{array}$
\end{tabular}


Tableau 3: Tests U de Mann-Whitney de l'expression des « sursauts » (SST).

\begin{tabular}{|c|c|c|c|c|c|c|c|c|c|c|}
\hline \multirow{2}{*}{$\begin{array}{l}\text { Temps } \\
\text { Comparaison }\end{array}$} & \multicolumn{2}{|c|}{$1 \mathrm{mn}$} & \multicolumn{2}{|c|}{$2 \mathrm{mn}$} & \multicolumn{2}{|c|}{$3 \mathrm{mn}$} & \multicolumn{2}{|c|}{$4 \mathrm{mn}$} & \multicolumn{2}{|c|}{$5 \mathrm{mn}$} \\
\hline & $\mathbf{U}$ & Sig & $\mathbf{U}$ & Sig & $\mathbf{U}$ & Sig & $\mathbf{U}$ & Sig & $\mathbf{U}$ & Sig \\
\hline SUB vs PRE & - & NS & - & NS & - & NS & - & NS & - & NS \\
\hline SUB vs INC & - & & 25 & 0,02 & 27,5 & 0,02 & 31 & 0,05 & 28 & 0,02 \\
\hline SUB vs NOU & - & & & NS & 37 & 0,05 & 36 & 0,02 & 29,5 & 0,02 \\
\hline SUB vs POS & - & & & NS & & NS & & NS & & NS \\
\hline PRE vs INC & - & & 66 & 0,02 & 80 & 0,05 & & NS & & NS \\
\hline PRE vs NOU & - & & 78,5 & 0,02 & 59 & 0,002 & 47 & 0,002 & 29 & 0,002 \\
\hline PRE vs POS & - & & & NS & 36 & 0,05 & 30 & 0,02 & 20,5 & 0,002 \\
\hline INC vs NOU & - & & 20,5 & 0,002 & 21,5 & 0,002 & 20,5 & 0,002 & 12 & 0,002 \\
\hline INC vs POS & - & & 16 & 0,002 & 14 & 0,002 & 13 & 0,002 & 8 & 0,002 \\
\hline NOU vs POS & - & NS & - & NS & - & NS & - & NS & - & NS \\
\hline
\end{tabular}

Sig.= significativité; $d d l=$ degré de liberté; $\mathrm{U}=$ valeur servant à calculer la probabilité $\mathrm{p}$ du test de Mann-Whitney; NS= non significatif.

Tableau 4: Tests de Kruskal-Wallis et de Mann-Whitney de l'évolution temporelle du «tissage » pour les trois catégoriesde femelles INC, NOU, POS.

\begin{tabular}{|c|c|c|c|c|c|c|c|c|c|c|}
\hline Temps & \multicolumn{2}{|c|}{$1 \mathrm{mn}$} & \multicolumn{2}{|c|}{$2 \mathrm{mn}$} & \multicolumn{2}{|c|}{$3 \mathrm{mn}$} & \multicolumn{2}{|c|}{$4 \mathrm{mn}$} & \multicolumn{2}{|c|}{$5 \mathrm{mn}$} \\
\hline \multicolumn{11}{|c|}{ Kruskal-Wallis, 2 ddl } \\
\hline $\mathrm{H}$ & \multicolumn{2}{|c|}{4,54} & \multicolumn{2}{|c|}{11,3} & \multicolumn{2}{|c|}{10,27} & \multicolumn{2}{|c|}{10,12} & \multicolumn{2}{|c|}{12,71} \\
\hline Sig & \multicolumn{2}{|c|}{ NS } & \multicolumn{2}{|c|}{$\mathrm{p}=0,007$} & \multicolumn{2}{|c|}{$\mathrm{p}=0,011$} & \multicolumn{2}{|c|}{$p=0,004$} & \multicolumn{2}{|c|}{$\mathrm{p}=0,003$} \\
\hline \multicolumn{11}{|c|}{ Mann-Whitney } \\
\hline Comparaison & $\mathbf{U}$ & Sig & $\mathbf{U}$ & Sig & $\mathbf{U}$ & Sig & $\mathbf{U}$ & Sig & $\mathbf{U}$ & Sig \\
\hline INC vs NOU & - & & 81 & 0,05 & 77 & 0,05 & 74 & 0,05 & 73 & 0,05 \\
\hline INC vs POS & - & & 15,5 & 0,002 & 22,5 & 0,02 & 19 & 0,02 & 17 & 0,002 \\
\hline NOU vs POS & - & & 41 & 0,05 & 40 & 0,05 & 34 & 0,02 & 34 & 0,02 \\
\hline
\end{tabular}

Sig.= significativité; $d d l=$ degré de liberté; $\mathrm{H}$ et $\mathrm{U}=$ valeurs servant respectivement à calculer la probabilité $\mathrm{p}$ des tests de Kruskal-Wallis et de Mann-Whitney; NS= non significatif.

\section{DISCUSSION}

Le fait que le cannibalisme des femelles pré-émergence envers les jeunes soit négligeable (Assi, 1996) et que la plupart des jeunes transportés (TRA) au cours des cinq minutes de confrontation ne soient pas systématiquement tués, laisse supposer que la réponse de la femelle à la présence des jeunes n'est pas a priori de les mettre à mort. C'est ce qui explique que certains jeunes saisis dans les chélicères (SAC), vraisemblablement de manière lâche, parviennent à s'échapper en stimulant avec leurs pattes antérieures la face ventrale de la femelle qui les relâche (REL) aussitôt.
Ces observations renforcent les précédents résultats par la confirmation des différences nettes entre les femelles en préémergence et en post-émergence (Assi et al., 1992 ; Assi et Horel, 1996 ; Assi, 1997). Ceci fait ressortir deux situations: l'une, reflétant une «attitude hostile» des femelles et l'autre, une «attitude tolérante». Par ailleurs, l'analyse de ces deux situations tend à montrer que les réponses des femelles dépendent aussi de l'attitude adoptée par les jeunes lors des confrontations; ceci vraisemblablement en relation avec l'intégration d'informations (attitudes comportementales notamment) émanant des jeunes. 


\section{Attitude hostile}

L'attitude hostile est observée lors des confrontations femelles en pré-émergence jeunes et se caractérise par les réactions de « sursauts » (SST) exprimées par les femelles. Ceci traduit un certain état de réactivité plus manifeste de ces femelles, les conduisant ainsi à rechercher activement les jeunes à l'occasion de leur moindre mouvement. Il est très rare de voir un jeune initier un contact physique en direction d'une de ces femelles.

Il est probable que l'expression de façon régulière des «sursauts" par les femelles agressives, traduise une inadéquation entre la présence des jeunes et l'état psychophysiologique des femelles susceptibles de les accepter, d'où le «transport» des jeunes par les femelles, probablement pour les éloigner de leur nid. Ainsi, ce «transport» de jeunes (TRA) ne peut être une attitude ou un comportement de type maternel; c'est-à-dire le comportement qu'une mère manifeste à l'occasion des soins qu'elle prodigue envers ses jeunes comme c'est le cas chez la rate quand elle ramasse ses jeunes qui s'éloignent du nid maternel (Beach et Jaynes, 1956a, 1956b; Deviterne et al., 1990 ; Kiss et al., 2012). On pourrait qualifier plutôt un tel comportement d'«attitude agressive de reconnaissance » : c'est à dire un comportement d'intolérance à l'égard de jeunes qui ne sont pas acceptés, donc plutôt rejetés, même s'ils sont spécifiquement reconnus par les femelles.

Par ailleurs, on ne peut pas non plus confondre un tel transport des jeunes avec celui d'une proie destinée à la consommation. L'étude de la prédation par les mères $C$. terrestris (Gundermann et al., 1989) a montré que les proies sont systématiquement ramenées dans le tube et fixées par plusieurs mouvements de rotation de la femelle autour de la proie. Ce type de comportement a été observé uniquement dans le cas des confrontations femelle-larves de grillon, dans ce cas, jamais un grillon n'a été transporté dans un coin sans être consommé (Assi, 1997).

\section{Attitude tolérante}

L'attitude tolérante est observée quand les jeunes sont en présence des femelles en post-émergence (NOU et POS). Elle se caractérise par d'intenses activités de «tissage» (TIS) des femelles. L'activité initiale de «sursauts» (SST) disparaît rapidement au profit du tissage. Au cours de ces confrontations, on a un nombre négligeable de femelles tueuses et de jeunes morts. Enfin, les contacts directs initiés par les jeunes en direction de chacune de ces femelles sont très fréquents.

\section{Problème d'intégration des informations émanant des jeunes}

Même si des cas rares de jeunes tués ou mangés par des femelles en pré-émergence sont enregistrés, la tendance générale de l'attitude de ces femelles est plutôt une intolérance qu'une absence de reconnaissance. Ici, les jeunes même reconnus en tant qu'homospécifiques, ne sont pas pour autant acceptés ou adoptés d'emblée. Cela pose probablement le problème de l'intégration des informations venant des jeunes par les femelles en pré-émergence.

Chez les espèces sociales, Krafft (1975) montre que, l'intégration de l'attitude comportementale d'un individu de la société par ses congénères, est l'un des principaux facteurs impliqués dans les processus de reconnaissance et de tolérance réciproque, limitant les morsures entre congénères. Ceci suggère que la façon dont se comportent les jeunes et l'interprétation qu'en fait la femelle, peuvent orienter les relations entre ces deux protagonistes, soit vers une attitude tolérante de la femelle, soit vers une attitude agressive de celle-ci vis-à-vis des jeunes. La réaction des femelles, en réponse à certaines attitudes comportementales adoptées par les jeunes lors des confrontations, semblent aller dans le sens de cette hypothèse. Ceci est mis en évidence par deux exemples de l'attitude 
comportementale des jeunes, caractéristiques des situations dangereuses: la fuite (FUI) du jeune à l'approche de la femelle et l'attitude de « soumission », c'est-à-dire l'immobilisation (IMO) instantanée du jeune lorsqu'il est contacté physiquement par une femelle. Dans ces conditions, les mécanismes de reconnaissance impliquant les composés ou phéromones cuticulaires chez les araignées (Trabalon et Assi, 2008; Trabalon et Bagnères, 2010; Grinsted et al., 2011) de même que, les indices génétiques sur quoi serait basée la différenciation entre parents et individus non apparentés chez Stegodyphus lineatus (Schneider et Bilde, 2008), sont des facteurs qui pourraient aussi probablement intervenir dans la régulations des interactions de confrontation entre les femelle et les jeunes conspécifiques.

\section{"L'immobilisation" (IMO) des jeunes}

Lorsqu'un jeune est contacté par une femelle, il s'immobilise aussitôt, ne faisant plus aucun mouvement; certains se faisant littéralement entraîner (ou rouler) par l'abdomen de la femelle lorsqu'elle passe audessus d'eux. Cette immobilisation instantanée des jeunes inhibe les femelles dans leur lancée agressive. Dans les conditions normales, les mères $C$. terrestris reconnaissent leurs petits à leur manière de se déplacer (Tretzel, 1961a, 1961b). Dans les conditions expérimentales réalisées ici, les jeunes sont introduits dans la boîte de la femelle sans ménagement afin de susciter une réaction rapide de la femelle. En plus, pour les femelles en pré-émergence, c'est leur première prise de contact avec des jeunes qui sont déjà à un âge de forte activité motrice; et pour les femelles en post-émergence, ces jeunes sont étrangers. Si l'on sait que la prédation est déclenchée par les vibrations inhabituelles des proies, l'immobilisation des jeunes devient nécessaire afin de limiter les attitudes agressives des femelles (particulièrement des femelles en préémergence) vis-à-vis d'eux.
Cette attitude comportementale d'immobilisation des jeunes est également mise en évidence chez les araignées sociales, A. consociata, lors de la chasse coopérative, où une araignée rattrapée par erreur par un congénère s'immobilise instantanément (Krafft, 1975).

D'autres travaux montrent également que, chez les fourmis ponérines, les ouvrières du genre Neoponera peuvent développer un rituel de soumission. $\mathrm{Au}$ cours de ce comportement, l'intruse se tasse sur le sol, prend l'aspect d'une nymphe dans son cocon. Cette posture inhibe non seulement l'agression de la résidente qui interrompt sa posture de piqûre, mais aussi déclenche chez la résidente une réaction de transport qui a pour but d'éloigner l'intruse du nid des résidentes et par conséquent d'épargner sa vie (Fresneau, 1963).

Dans la présente étude, ce type de comportement est enregistré surtout avec les femelles en pré-émergence. Ainsi, des jeunes dans leur position d'immobilisation sont transportés passivement après quelques tapotements et sont déposés au fond de la galerie. La plupart des jeunes ainsi traités ne sont pas tués. Par contre, la «turbulence » des jeunes au moment où ils sont contactés par la femelle provoque immédiatement des interactions brutales. Ces interactions se terminent souvent par une saisie ferme du jeune dans les chélicères de la femelle et peut conduire à sa mise à mort.

\section{La "fuite" (FUI) des jeunes}

La plupart des jeunes fuient systématiquement à l'approche de la femelle ou sous l'action d'une attaque même à distance de celle-ci. Par cette attitude, les jeunes échappent facilement à la femelle qui serait tentée de les agresser. Les risques de rencontres avec une femelle sont d'autant plus réduits que les jeunes, après leur fuite, vont se camoufler derrière les structures soyeuses, se mettant ainsi à l'abri de tout contact direct avec la femelle. 
Ce comportement de fuite avec camouflage des jeunes, caractéristique des interactions jeunes-femelles en préémergence, est également observé avec les jeunes lors de la capture d'une proie par leur mère. Dans ce cas, les jeunes, en se mettant à l'abri derrière les structures soyeuses, non seulement facilitent la capture de la proie par leur mère, mais aussi évitent de se faire attaquer par erreur. En effet, chez $C$. terrestris, l'activité prédatrice de la mère demeure intacte et s'accroît même au cours du développement des jeunes (Gundermann et al., 1989). De ce fait, l'identification formelle des jeunes par la mère nécessite un contact direct surtout en situation inhabituelle. Ainsi, l'attitude de soumission adoptée par les jeunes lorsqu'ils sont contactés par les femelles et les informations tacto-chimiques recueillies par celles-ci, permettent leur identification (Assi, 1997).

Il convient de remarquer que si l'immobilisation des jeunes intervient probablement pour atténuer l'élan agressif des femelles en pré-émergence, elle ne semble pas, par contre, supprimer totalement le conflit que ces jeunes «indésirés» engendrent par leur présence, puisqu'ils sont saisis et expulsés par ces femelles, en dépit de la posture qu'ils adoptent. L'absence des saisies et des transports de jeunes par les femelles en post-émergence, malgré les contacts actifs, est une indication de l'intégration des relations mère-jeunes. Ce qui n'est pas le cas des femelles en préémergence, chez lesquelles les saisies et le transport des jeunes sont fréquents; ces femelles doivent nécessairement passer par une période d'adaptation.

\section{Conclusion}

L'expression de "sursauts" traduit vraisemblablement une attitude agressive des femelles vis-à-vis des jeunes, car ce sont les femelles en pré-émergence ou femelles "tueuses" qui présentent significativement la manifestation de ce comportement. L'activité de tissage peut-être au contraire qualifiée de comportement maternel, son expression semble traduire une certaine tolérance et/ou reconnaissance des femelles vis-à-vis des jeunes. En effet, ce sont les femelles en postémergence déjà montrées comme les plus tolérantes qui présentent les principales caractéristiques du tissage.

\section{REMERCIEMENTS}

Nous exprimons notre gratitude aux Professeurs Trabalon M, Doh KP, Bohui H et aux quatre référés anonymes pour leurs utiles suggestions pour améliorer la qualité de cette étude.

\section{REFERENCES}

Assi BD, Horel A ,Gundermann JL. 1992. Onset and maintenance of maternal prey_sapply activity in the spider Colotes terrestris (Araneae, Agelenidae). Behaviour, 120: 1-10.

Assi BD, Horel A. 1996. Social-maternal relations in Coelotes terrestris, (Araneae, Agelenidae): Influence of the female reproductive state on its tolerance towards conspecifi c spiderlings. Behaviour Processes, 36: 19-26.

Assi BD. 1997. Intraspecific indentification and tolerance in the social maternal behaviour of Coelotes terrestris (Araneae, Agelenidae). Behav. Process, 39: 231-239.

Assi BD, Trabalon M. 2005. Régulation du comportement maternel chez l'araignée Coelotes terretris (Aranaea, Agelenidae): Influence de l'âge des juvéniles sur la tolérance des femelles. BIOTERRE, Rev. Inter. Sci. Vie Terre, 5: 18-30.

Beach FA, Jaynes J. 1956a. Stadies of maternaI retrieving in Rats: Recognition of young. J. Mammal, 37: 177-180.

Beach FA, aynes J. 1956b. Stadies of maternaI retrieving in Rats: Sensory aras involved in the lactating femaIe respoo.se 10 her yooog. Behaviour, 10: 104-125. 
Billick I, Weidmann M, Reithel J. 2001. The relationship between ant-tending and maternal care in the treehopper Publilia modesta. Behav. Ecol. Sociobiol., 51: 41- 46.

Chatfield CH, Lemon RE. 1970. Analysing sequences of behavioural events. $J$. Theor. Biol., 29: 427-445.

Deleurance S. 1957. Contribution à l'étude biologique des Polistes (Hyménoptères Vespidés) L'activité de construction. Ann. Soc. Nat., Zool., 19(11): 91-222.

Deviterne D, Desor D, afft B. 1990. Maternal behavior variations and adaptations, and pup development within litters of various sizes in Wistar Rat. Dev. Psychobiol., 23(4): 3-49-360.

Eickwort GG. 1981. Presocial lnsects. In Social Insects, Hennann HR (ed). Academie Press: New York; 199-280

Fresneau D. 1963. Fermeture des sociétés chez les fourmis primitives (La). Film: Réalisation: Wauquier P. Production et Distribution: S.F.R.S.

Gervet J. 1964a. La ponte et sa régulation dans la société polygyne de Polistes gallicus L. Ann. Sei. Nat. Zool., 6(12): 601-778.

Gervet J, Truc C. 1976. Le déroulement du comportement durant le cycle nidificateur de l'ammophile Podalonia hirsuta Scopoli. Netherlands J. Zool, 26(3): 319-382.

Grasse PP. 1942. L'essaimage des termites. Essai d'analyse causale d'un complexe instinctif. Bull. Biol., 76: 347-382.

Grinsted L, Bilde T, d'Ettorre P. 2011. Cuticular hydrocarbons as potential kin recognition cues in a subsocial spider. Behavioral Ecology, 22: 1187-1194.

Gundermann JL, Horel A, Krafft B. 1989. Maternal food-supply activity and its regulation in Coelotes terrestris (Araneae, Agelenidae). Behaviour, 107 : 278-296.

Horel A, Gundermann JL. 1992. Egg sac guarding by the funnel-web spider
Coelotes terrestris: function and development. Behav. Process, 27: 85-94.

Kiss ACI, Woodside B, Felicio LF, AnselmoFranci J, Damasceno DC. 2012. Impact of maternal mild hyperglycemia on maternal care and offspring development and behavior of Wistar rats Physiology \& Behavior, 107: 292-300.

Krafft B. 1970. Contribution à la biologie et à l'éthologie d'Agelena consociata, Denis. (Araignée sociale du Gabon) Ill. Elude expérimentales de certains phénomènes sociaux. Biol. Gabon, 8 : 3-56.

Krafft B. 1975. Proc. 6th La tolérance réciproque chez l'Araignée sociale Agelena consociata Denis. Proc. 6th Int. Arachnol. Congr., Amsterdam : 107-112.

Park Y, Choe J. 2003a. Territorial behavior of the Korean wood-feeding cockroach, Cryptocercus kyebangensis. Journal of Ethology, 21: 79-85.

Park YC, Choe JC. 2003b. Effects of parental care on offspring growth in the Korean wood-feeding cockroach, Cryptocercus kyebangensis. Journal of Insect Physiology, 21: 71-77.

Salomon M, Schneider J, Lubin Y. 2005. Maternal investment in a spider with suicidal maternal care, Stegodyphus lineatus (Araneae, Eresidae). Oikos, 109: 614-622.

Schneider JM, Bilde T. 2008. Benefits of cooperation with genetic kin in a subsocial spider. Proceedings of the National Academy of Sciences of the United States of America, 105: 1084310846.

Stefani V, Del-Claro K, Silva LA, Guimaraes B et Tizo-Pedroso E. 2011a. Mating behaviour and maternal care in the tropical savanna funnel-web spider Aglaoctenus lagotis Holmberg (Araneae: Lycosidae). Journal of Natural History, 45: 1119-1129.

Trabalon M, Assi BD. 2008. Effets of web chemical signatures on intraspecific recognition in subsocial spider, Coelotes 
terrestris (Araneae). Animal Behaviour, 76: 1571-1778.

Trabalon M, Bagnères AG. 2010. Contact recognition pheromones in spiders and scorpions. In Insect Hydrocarbons: Biology, Biochemistry, and Chemical Ecology, Blomquist GJ, Bagnères AG (eds). Cambridge University Press: Cambridge; 344-374.

Tretzel E. 1961a. Biologie. Oekologie und Brutpflege von Coelotes terrestris (Wider) (Araneae, Agelenidae).1 Biologie und Oekologie. Z. Morph. Oekol. Tiere, 49: 658-745.

Tretzel E. 1961b. Biologie. Oekologie und Brutpflege von Coelotes terrestris
(Wider) (Araneae, Agelenidae). II Brutpfiege. Z. Morph. Oekol. Tiere., 50: 375-542.

Viera C, Ghione S, Costa FG. 2006. Regurgitation among penultimate juveniles in the subsocial spider Anelosimus cf. studiosus (Theridiidae): are males favored? Journal of Arachnology, 34: 258-260.

Viera C et Romero GQ. 2008. Maternal care in a neotropical jumping spider (Salticidae). Journal of Zoology, 276: 237-241.

Yip EC, Rayor LS. 2014. Maternal care and subsocial behavior in spiders. Biological Reviews, 89: 427-449. 